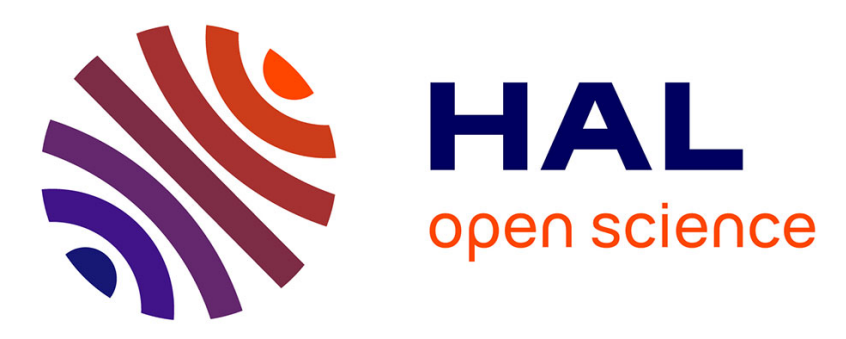

\title{
Analysis of necking in high speed experiments by stereocorrelation
}

Gilles Besnard, François Hild, Jean-Michel Lagrange, Philippe Martinuzzi, Stéphane Roux

\section{- To cite this version:}

Gilles Besnard, François Hild, Jean-Michel Lagrange, Philippe Martinuzzi, Stéphane Roux. Analysis of necking in high speed experiments by stereocorrelation. International Journal of Impact Engineering, 2012, 49, pp.179-191. 10.1016/j.ijimpeng.2012.03.005 . hal-00722694

\section{HAL Id: hal-00722694 \\ https://hal.science/hal-00722694}

Submitted on 3 Aug 2012

HAL is a multi-disciplinary open access archive for the deposit and dissemination of scientific research documents, whether they are published or not. The documents may come from teaching and research institutions in France or abroad, or from public or private research centers.
L'archive ouverte pluridisciplinaire $\mathbf{H A L}$, est destinée au dépôt et à la diffusion de documents scientifiques de niveau recherche, publiés ou non, émanant des établissements d'enseignement et de recherche français ou étrangers, des laboratoires publics ou privés. 


\title{
Analysis of necking in high speed experiments by stereocorrelation
}

\author{
Gilles Besnard, ${ }^{a, b}$ François Hild, ${ }^{a, d}$ Jean-Michel Lagrange, ${ }^{b}$ \\ Philippe Martinuzzi, ${ }^{c}$ Stéphane Roux ${ }^{a}$ \\ (a): Laboratoire de Mécanique et Technologie (LMT-Cachan) \\ ENS Cachan / CNRS / UPMC / PRES UniverSud Paris \\ 61 Avenue du Président Wilson, F-94235 Cachan Cedex, France \\ (b): CEA, DAM, DIF, F-91297 Arpajon, France \\ (c): CEA, DAM, Valduc, F-21120 Is sur Tille, France \\ (d): corresponding author.
}

\begin{abstract}
Tensile tests with Hopkinson bars are monitored with high speed cameras to extract 3D displacement fields and the corresponding strain fields by resorting to stereocorrelation. The aim of the experiments is to analyze the onset and development of necking for various strain rates and samples. Various challenges have to be dealt with, namely small definition of the pictures, large displacements that occur during the experiment, and the need for good spatial resolutions to capture the strain levels within the neck. In order to address all these issues, pre-correction techniques are used to allow for the required stability and accuracy of the analysis.

Eight experiments on cylinders and parallelepipeds at different loading rates are analyzed. Either single or multiple neck inceptions were observed, irrespective of the mean strain rate, supporting a random nucleation picture rather than a deterministic
\end{abstract}


result of the constitutive law. Local strains up to about $600 \%$ were measured. In the case of multiple necking, a selection leading to the growth of a dominant one is observed, in agreement with Mott's theory of an "unloading wave" emanating from neck inception. A propagation speed of this wave can be estimated from the analysis and is shown to agree with the theoretical prediction.

Key words: Digital Image Correlation, Full-field measurements, Necking, Spatial registration, Stereocorrelation, Temporal registration.

\section{Introduction}

The experimental analysis of necking under dynamic loading conditions mostly relies on expanding metal rings by electromagnetic loading [1-3] for strain rates on the order of $10^{4} \mathrm{~s}^{-1}$. Multiple necking was observed along the circumference of the tested rings. Further, the number of necks (and fragments) and the failure strain increase with the expansion velocity. In all the analyses, the data concern macroscopic quantities such as the fragment number, the expansion velocity. In the following, it is proposed to study necking for strain rates on the order of $10^{3} \mathrm{~s}^{-1}$ so that high speed cameras can be used to monitor experiments in split Hopkinson pressure bars. Based on the pictures, quantitative analyses can be conducted in terms of surface shape and strain field.

Split Hopkinson pressure bars [4,5] are a classical testing device to analyze

Email addresses: gilles.besnard@lmt.ens-cachan.fr (Gilles Besnard, $\left.{ }^{a, b}\right)$, francois.hild@lmt.ens-cachan.fr (François Hild, $\left.{ }^{a, d}\right)$, jean-michel.lagrange@cea.fr (Jean-Michel Lagrange $\left.{ }^{b}\right)$, philippe.martinuzzi@cea.fr (Philippe Martinuzzi, ${ }^{c}$ ), stephane.roux@lmt.ens-cachan.fr (Stéphane Roux $\left.{ }^{a}\right)$. 
the behavior of materials under dynamic loading conditions. In most cases, the load and mean strain experienced by samples are extracted from the analysis of the signals delivered by strain gauges glued on the input and output bars (i.e., usually quite far from the sample itself). More recently, with the development of (stereo)correlation techniques [6,7], it is possible to measure 3D displacement and 2D strain fields. When applied to pictures acquired by high speed cameras, the previous kinematic data are measured directly on the sample surface [8]. These techniques then allow for various studies to be performed to monitor high speed experiments. For instance, Barthelat et al. [9] analyze torsion tests on nanocrystalline coatings of tungsten carbide-cobalt cermets, Elnasri et al. [10] compression tests on aluminum and nickel foams, Gilat et al. [11] tensile tests on copper samples, and Besnard et al. [12] necking in tantalum. Kurigulige et al. [13] follow crack propagation of an impacted sample. Tiwari et al. [14] and Besnard et al. [15] analyze experiments during during blast loadings.

In most of the above mentioned cases, the measured strain levels do not exceed 10\%. The experiments reported herein aim at monitoring the inception and development of necking. This analysis therefore requires to measure strain levels well above $100 \%$. The aim of the present study is to show that such measurements are feasible, even at quite high strain rates, which imply dealing with low definition pictures (typically of the order of a few tens of kpixels). Once these results are available, the next step will consist in comparing them with finite element simulations, which are not presented herein.

The paper is organized as follows. First, the experimental configuration is introduced. Second, the stereocorrelation technique implemented herein uses space and time registrations based upon a global approach to digital image cor- 
relation [15]. New initialization procedures need to be implemented to measure large displacements and large strains. Third, the results of the stereocorrelation analysis are presented to analyze necking in pure aluminum.

\section{High speed experiments}

The tested samples are either cylinders $5 \mathrm{~mm}$ in diameter, or parallelepipeds with a height of $8 \mathrm{~mm}$ and a width of $2.5 \mathrm{~mm}$. Both geometries have gauge lengths varying from 10 to $40 \mathrm{~mm}$ (Figure 1). Their ends are threaded so that they can be fixed on the input and output bars end. Different gauge lengths are tested to investigate size effects on the occurrence of multiple necking. Pure aluminum was chosen in the tests reported herein because of its very high ductility. Its Young's modulus is equal to $69 \mathrm{GPa}$, Poisson's ratio is equal to 0.33 , and its density is equal to $2,700 \mathrm{~kg} \cdot \mathrm{m}^{-3}$. In the present case, the tensile loading is created by the impactor that hits the shoulder of the input bar (Figure 2). The input bar then applies the load to the sample as in any classical configuration. The relative motion between the two bars allows the load to be applied to the sample.

A stereovision setup consisting of two identical PHOTRON APX RS fast cameras is used to determine the 3D shape of the external surface and the corresponding 3D displacements. All optical devices (e.g., lenses, supports) are identical for both cameras. Figure 1(a) shows the stereovision setup for which special attention was paid to lighting conditions. The two cameras are connected together and electronically synchronized so that pictures are acquired with a time delay less than a few $100 \mathrm{~ns}$ [16]. To protect the objective lenses from heat, a screen is put below them. For the eight tests presented 
herein, the frequency of acquisition varies between 45,000 and 52,500 fps with a (low) definition ranging from 37 to 57 kpixels (Tables 1 and 2). The definition / acquisition rate is the result of a compromise so that enough pictures are available to perform a successful registration to analyze necking in samples with very small diameters.

Because of the chosen picture definition and acquisition rate, the displacement amplitudes between two consecutive pictures will be large and special pre-correction procedures are called for to allow the spatial and temporal registrations to be successful when using a global approach to DIC [15] applied to cylinders.

\section{Stereocorrelation applied to high speed experiments}

Extraction of a 3D shape is performed through stereovision exploiting at least two view points [7]. When applied at different instants of time, it also possible to measure 3D surface displacements by stereocorrelation (or 3D-DIC), and thus the components of the displacement gradient in the tangent plane of any point of the observed surface. In the following analyses, a global approach to stereocorrelation will be used. It consists in using finite element meshes with 4-noded quadrilateral elements (i.e., Q4-DIC [20]). Consequently, the spatial and temporal registrations will be performed with Q4-DIC. The interested reader will find details on the practical implementation of the 3D-DIC code in Ref. [15]. The Q4-DIC procedure rests on multi-resolution analyses in which successive linearizations and corrections are performed via a modified Newton algorithm [20]. In the present case, the image definition is small so that very few scales can be used. Consequently, another route has to be followed when 
complex shapes (e.g., cylinders) are studied. It is based on the initialization of the correlation procedure.

For the two (spatial and temporal) registrations, the problem to be solved is of a different nature. For the spatial registration, the main challenge is related to the fact that the diameter of the sample is small so that large deformations occur when the image pairs are to be registered to reconstruct the 3D shape. For the temporal registration, large displacements and strains occur between two consecutive instants of time so that the coarse-graining technique cannot be applied as usual [20] since the definition of the pictures is too small. Consequently, two different procedures are developed.

\subsection{Initialization of the spatial registration}

The aim of this first pre-correction is to increase the surface of cylinders that can be monitored by stereovision, and the quality of the initial reconstruction. It consists in the evaluation of the low frequency components of the displacement fields. The latter ones have a large amplitude. When evaluated, the pictures are pre-corrected so that the classical registration techniques will be successful since the subsequent corrections are small in amplitude. It is also based on the a priori knowledge of the sample geometry that has, as a first approximation, a symmetry of revolution.

The very first reconstruction is performed without any information. It is restricted to a small part of the picture where the deformations are not too large. From the reconstructed shape, a cylinder is sought that interpolates best, in the least squares sense, the cloud of reconstructed points. From this information, it is possible to compute for any point of the surface, its location in the 
pictures acquired by both cameras as shown in Figure 3. This projection gives the point locations and the displacement to apply to predict where any point in the picture of one camera has moved in the picture of the other one.

This first estimate is used as initialization of the global approach. Because of the curvature of the object, the apparent displacement amplitudes are very important on the edges of the picture and very small in the central part. When the pre-correction procedure is used, this effect is accounted for and the corrections are almost uniform in amplitude over the whole region of interest. This makes the registration much easier and more robust. The effect of this pre-correction is to increase the surface that can be reconstructed as shown in Figure 4. A 50\% increase of the reconstructed surface is obtained and the root mean square difference between the reconstructed surface and a perfect cylinder is equal to $41 \mu \mathrm{m}$ prior to pre-correction, and $19 \mu \mathrm{m}$ afterward. This quantity is comparable in magnitude to the roughness of the reconstructed surface.

For parallelepipedic samples, the present procedure was not used since the shape of their external surfaces is easier to register. As such, the present precorrection does not apply to the temporal registration for both sample series. Another procedure will be followed.

\subsection{Initialization of the temporal registration}

The main objective is to decrease the reconstruction uncertainty by precorrecting the large displacements that occur between consecutive pictures. Two routes can be followed. The first one consists in updating the reference picture so that large displacements can be measured by considering a series 
of pictures [21,17], namely, the reference picture of the current analysis is the deformed one of the previous step. However, the larger the number of pictures, the larger the measurement uncertainty for the pictures as their number increases (i.e., the measurement uncertainties are gradually cumulated since the reference picture is updated in the analysis). The second is to use a first estimate as an initialization of the temporal registration in the longitudinal direction. This initial estimate, which corresponds to the result of the analysis corresponding to route no. 1 , no longer requires to update the reference picture. Otherwise, the number of measurement points decreases dramatically because of the low definition of the pictures.

In the transverse direction, a contour-detection technique (i.e., based upon thresholded gradients) is used and coupled with the evaluation of the axis of the cylinder, which was determined for the first 3D shape. From these two quantities, a first estimate of the radial displacement is obtained. When the initial (Figure 5(a)) and current (Figure 5(b)) contours are compared, transverse motions are estimated. The transverse displacement is assumed to keep the geometry axisymmetric so that from the analysis of a single contour it is possible to evaluate the displacement of any point of the external surface. For parallelepipedic samples the transverse direction is treated in the same way as the longitudinal direction. With these initial guesses, the correlation algorithm is run and only consists in correcting for small deviations induced by the various hypotheses made during the initialization step. In particular, no image updating is needed when a series of temporal analyses has to be carried out.

The second advantage of the proposed strategy is related to the elements used in the Q4-DIC approach. Their sizes could be decreased thereby allowing for 
a richer analysis, in particular within the necks. An example of picture "stabilization" is shown for cylinder A in Figure 6(e) when compared with the raw information (Figure 6(c)) and with the reference picture (Figure 6(a)). The corresponding longitudinal and transverse displacements are shown in Figure $6(\mathrm{~b}, \mathrm{~d})$. The range of longitudinal displacements is of the order of 100 pixels, which is very large when considering only 15 pictures with a low definition. The transverse displacements are more modest in amplitude, but the main difficulty is related to the shape of the surface. Had this initialization step not been considered, the analysis would not have converged.

In the following section, necking under dynamic loading condition will be analyzed by using the procedure developed herein.

\section{Experimental results}

In the following study, the size of the elements used in Q4-DIC to register spatially and temporally the different pictures is chosen to be equal to 8 pixels. This size is a compromise between the measurement resolution and the spatial resolution [20]. The latter is critical here since low definition pictures are used (Tables 1 and 2).

\subsection{Analysis of the 3D shape of samples with necks}

The 3D shape of cylinders is obtained by using the following steps:

- Reconstruction of the surface in its reference configuration. The spatial registration makes use of the above discussed pre-correction procedure. 
- Extraction of the cylinder axis by fitting, in the least squares sense, the reconstructed shape with a cylinder.

- Evaluation of the distance of each 3D point with respect to the cylinder axis, and the corresponding radius.

- Estimation of the average radius in each plane perpendicular to the cylinder axis.

For the parallelepipedic samples, the shape of the external surface does not require any special treatment when the temporal pre-correction procedure described above is utilized.

The mean reconstruction uncertainty is of the order of $30 \mu \mathrm{m}$ for 8-pixel elements and all the experiments reported hereafter. It was evaluated by propagating the registration uncertainty for the reconstruction process [22]. The reason for this high value is due to the small definition of the images so that the physical size of one pixel varies between 50 and $83 \mu \mathrm{m}$ (Table 1 and 2), and the small element size (i.e., the smaller the element size, the larger the uncertainty associated with the correlation procedure [20]). It is worth noting that thanks to the pre-correction techniques introduced previously, the uncertainty level is independent of the number of analyzed pictures since there is no need to update the reference picture.

Figure 7 shows the reconstructed outer surface for the four tested cylinders at the end of each image sequence. In the same figure, the pictures recorded by the left camera are also shown for comparison purposes. Even though the strains of the cylinders are very high in the neck, it is possible to follow their development with the correlation procedure adapted to low definition pictures. This is particularly the case for samples $\mathrm{C}$ and $\mathrm{D}$ for which only 64 pixels were available in the height of the picture (i.e., a very small angular part was visible 
by both cameras). For the parallelepipedic samples, the picture recorded by the right camera is shown for times close to fracture in Figure 8. The longitudinal strain fields are also given. All necks are visible.

To follow the development of necking in cylinders, Figure 9 shows the change of the radius profile with the picture number. As the definition of the pictures decreases (i.e., from samples A to D), the uncertainty level in the reconstruction increases (i.e., the apparent roughness increases from samples A to D). This effect is due to the decrease of the angular part that is monitored. From these data, necking is clearly revealed, and leads to radial variations of more than $1.3 \mathrm{~mm}$ to be compared with an initial radius on the order of $2.5 \mathrm{~mm}$. It is possible to monitor the formation of necks by analyzing the longitudinal profile of the mean radius. At the beginning of the experiment, the radius decreases uniformly along the cylinder axis. Then, the profile becomes less uniform and one (samples A and D) or possibly two necks (samples B and C) are formed.

\subsection{Loading condition}

Apart from the change in the reconstructed 3D shape, it is possible to use the 3D displacement fields. Their mean component at both ends of the analyzed region of interest allows us to evaluate the mean longitudinal strain, which was checked to coincide to within measurement uncertainties with the mean major principal strain $\left\langle\epsilon_{2}\right\rangle$. Figure 10 shows the change of the latter with time for the four tested cylinders. For each of the four tests, a virtually constant strain rate is observed, even after the first plateau. From these data it can be concluded that the mean strain rates vary from $1600 \mathrm{~s}^{-1}$ (sample A) to 
$600 \mathrm{~s}^{-1}$ (sample D). It is worth noting that even though the strains localize within the neck, the mean strain rate remains virtually constant as a result of the impactor traveling at a constant velocity (Table 3). In the Appendix, it is shown that these trends can be understood from the Lagrange diagram of the setup and the different states in the bars and the sample. The same trend is observed for the four parallelepipeds (Figure 11). The mean strain rates vary from $1400 \mathrm{~s}^{-1}$ (sample E) to $600 \mathrm{~s}^{-1}$ (sample H), see Table 4. These results indicate that the experiments reported herein were strain rate controlled on a macroscopic level.

\subsection{Strain fields}

The strains are computed in the tangent plane of each considered surface point. Because of the extreme conditions encountered herein (especially with cylinders), for instance the radius variations along the longitudinal axis (Figure 9), the strain fields that are derived from the projection of the displacement onto the local tangent plane will be fluctuating more than usual. Further, in the case of large transformations, special care has to be exercised. Experimentally, only in-plane components of the deformation gradient $\mathbf{F}$ are accessible. Consequently, some additional hypotheses are to be made to evaluate large strains. In the present case, nominal (or Cauchy-Biot) strains are computed. It is assumed that the normal vector to the surface is an eigen direction. In each element $\Omega_{e}$, the mean in-plane deformation gradient $\mathbf{F}_{p}$ reads

$$
\mathbf{F}_{p}=\mathbf{1}_{p}+\int_{\partial \Omega_{e}} \mathbf{u}_{p} \otimes \mathbf{p} \mathrm{d} s
$$

where $\mathbf{1}_{p}$ is the in-plane unit tensor, $\mathbf{u}_{p}$ the in-plane projection of the $3 \mathrm{D}$ displacement vector $\mathbf{u}, \mathbf{p}$ the outward normal to the element edges $\partial \Omega_{e}$ lying 
on the surface tangent plane, and $s$ the curvilinear abscissa. The mean inplane nominal strain tensor $\boldsymbol{\epsilon}_{p}$ is computed by considering the in-plane right stretch tensor $\mathbf{S}_{p}$ associated with the polar decomposition of $\mathbf{F}_{p}$

$$
\boldsymbol{\epsilon}_{p}=\mathbf{S}_{p}-\mathbf{1}_{p}
$$

In the present case, the strain resolution is of the order of $1 \%$. This value was determined by analyzing the very first pictures for which no loading was applied. This value degrades as the shape of the reconstructed surface becomes noisier.

With the reported strain rates (Tables 3 and 4 ) and the integration time of each experiment (Tables 1 and 2), it is concluded that the mean strain increment during picture acquisition is less than $1 \%$ (i.e., the estimated strain resolution). Consequently, even though strain rates larger than the mean value occur in the necks, it is believed that the integration time remains small enough not to bias the strain measurements.

As for the reconstructed surface, only the mean major strain $\epsilon_{2}$ for each longitudinal position is analyzed. Figures 12 and 13 show the major strain profiles for the same pictures as those shown in Figures 9 for the cylinders. From these results it can be seen that the strains are mostly concentrated in necks after their inception and their levels significantly lower elsewhere. For sample A, the mean major strain is equal to $52 \%$, and the strains in the neck exceed $180 \%$. For sample B, the strain levels in the neck reach values greater $300 \%$. For samples $\mathrm{C}$ and D, the strains become even higher to reach $600 \%$. All these values are achieved by using at most $2 \times 55$ pictures in the correlation analysis. For the parallelepipedic samples, the strain fields at the end of the load history are shown in Figure 8. Very high strain levels are observed and 
the presence of one or two necks is observed.

In Figures 12 and 13, the estimate of the neck length is depicted. Its physical size is given in Tables 3 and 4 . It varies between $1.3 \mathrm{~mm}$ (sample B) and $2.7 \mathrm{~mm}$ (sample F). No clear dependence with the strain rate is observed. The comparison between the mean major strain and the average value in the neck(s) is shown in Figures 14 and 15. From these data, the onset of localized necking is determined by looking for the instant when both curves depart from each other. It is also worth noting that in all cylinder-shaped specimens where two necks appear, the first to initiate is never the one that will become dominant. This conclusion is different for parallelepipeds. In all cases where two necks appear, only one eventually develops. The strain levels corresponding to the inception of the dominant neck are reported in Tables 3 and 4 . No clear conclusion can be drawn from the analysis of the results in terms of strain rate dependence. It is worth remembering that the underlying elasto-plastic behavior of aluminum itself is, as a first approximation, strain-rate independent [23]. Conversely, it can be concluded that the inception strain is a random variable with a rather large scatter. Such an observation could support the hypothesis that neck inception is controlled by initial geometric imperfection that cannot be resolved, rather than being dictated by the constitutive law.

\subsection{Analysis of multiple necking}

On the picture of the deformed configuration (Figure $7(\mathrm{~b})$ ) and on the longitudinal strain fields (Figure $8(\mathrm{~b}, \mathrm{~d})$ ) two necks are visible at the end of the experiments. The aim of the following analysis is to understand the history of the formation of these two necks. With these data, it is possible to follow the 
change of the mean longitudinal strain as a function of time and space, and therefore to monitor the formation of necks by analyzing the corresponding profiles. The time evolution of the measured profiles is shown in Figure 9(b). At the beginning of the experiment, the radius reduction is uniform along the cylinder axis. From picture no. 21 and on, the profiles become less uniform. For picture no. 31, two necks are clearly formed and have about the same size. After image no. 31, one neck continues to develop while the other one has stopped growing. The former will eventually lead to the failure of the sample.

With Figure 14(b), it is possible to conclude that neck inception did not occur at the same time, and that one of the necks concentrated the strains very early on after their joint formation. For sample B, the two necks could grow during about $300 \mu \mathrm{s}$, and the distance between the two necks is equal to $9.5 \mathrm{~mm}$. Similarly, from Figure 15(b,d), the time duration of both neck growths is equal to $250 \mu$ s for sample $\mathrm{F}$, and $500 \mu$ s for sample H. The neck separation is approximately equal to $8.5 \mathrm{~mm}$ for sample $\mathrm{F}$ and $24 \mathrm{~mm}$ for sample $\mathrm{H}$.

The previous analysis can be discussed in terms of Mott's model [24]. When multiple necks form, the regions connecting them do not yield but gradually unload. Each neck behaves independently as long as the information about unloading has not reached other necks. This information is carried by an "unloading wave" travelling at a speed of $v_{y}$. The unloading wave caused by the first formed neck will reach its neighbors and stop their growth before the inverse interaction occurs. In the present case, $v_{y} \approx 40 \mathrm{~m} / \mathrm{s}$. This value is obtained from the above analysis. If the "unloading wave" is assumed to propagate at a speed of $\sqrt{\sigma_{y} / \rho}[25,26]$, and when $\sigma_{y} \approx 5 \mathrm{MPa}$ for pure aluminum, a value $v_{y} \approx 43 \mathrm{~m} / \mathrm{s}$ is found. This result therefore validates the applicability of Mott's model. However, the fact that the first neck does not become domi- 
nant is related to the particular loading conditions of split Hopkinson bars for which constant strain rate regimes are separated by a constant strain regime (Figures 10 and 11).

\section{Summary}

A stereocorrelation technique was applied to the analysis of necking in high speed tensile tests. The spatial and temporal registration is based on a global approach to digital image correlation using Q4 elements. The low definition of the pictures due to the high acquisition frequency required pre-corrections of the temporal registration to capture large displacements and strains in cylinders. An additional pre-correction procedure of the spatial registration was needed since the radius of the sample was small. Both pre-correction techniques allowed for the reconstruction of 3D shapes in a large part of the external surface of the sample, and the measured strain levels was shown to exceed $600 \%$ in the dominant neck prior to final failure by using 8-pixel Q4 elements and $2 \times 55$ pictures.

Some additional gain to the previous results can be obtained by still improving the temporal registration. To decrease the measurement uncertainties, it is possible to resort to a spatiotemporal registration in which the displacement field is not only decomposed over a spatial basis, but also a temporal one. The early results indicate that such approaches are able to capture localized phenomena $[12,27]$. Their extension to stereocorrelation is currently implemented and will be tested.

By following the change of the surface and longitudinal strain profiles, the inception strain of necks is determined. In some cases two necks are formed and 
one of the necks is shielded by the other one, thereby interrupting its further growth. Only one neck is fully developed in all the experiments on cylinders reported herein. For long parallelepipeds two necks could grow simultaneously for a longer time. This result shows that neck formation is the result of a competition between multiple neck inceptions and the growth of dominant necks that obscure others as proposed by Mott [24]. The analysis of strain fields shows that the local levels are significantly larger than the global ones. This is to be expected from any localized phenomenon such as necking. In the present case, a ratio of up to 15 is found between local and global strain levels.

When analyzing the dominant neck of the eight experiments reported herein, it is not possible to conclude about a strain rate sensitivity of their inception strain. The same conclusion could be drawn when the neck width was analyzed. Conversely, the inception strain itself is very scattered, and calls for a probabilistic treatment.

Last, these results are very useful to validate constitutive models describing necking. Moreover, the measured end displacements of the region of interest can be used as boundary conditions to the numerical simulations so that there is no need to model the complete split Hopkinson bar setup, but only a very small region of the sample. Further, the strain heterogeneities during the whole test were monitored and constitute a discriminating benchmark test for numerical modeling.

\section{Acknowledgments}

The authors wish to thank Damien Delaplanche, Nicolas Granier, and Gilles Roy of CEA Valduc, Stéphane Pattofatto and Han Zhao of LMT-Cachan 
for the discussions about the experiments reported herein. This work was supported by a grant from Région Île-de-France. 


\section{References}

[1] I. M. Fyfe and A. M. Rajendran, Dynamic pre-strain and inertia effects on the fracture of metals, J. Mech. Phys. Solids 28 (1980) 17-26.

[2] D. E. Grady and D. A. Benson, Fragmentation of metal rings by electromagnetic loading, Exp. Mech. 12 (1983) 393-400.

[3] M. Altynova, X. Hu and S. Daehn, Increased ductility in high velocity electromagnetic ring expansion, Metall. Mat. Trans. 27A (1996) 1837-1843.

[4] D. Hopkinson, A Method of Measuring the Pressure Produced in the Detonation of High Explosives or by the Impact of Bullets, Phil. Trans. Roy. Soc. A 213 (1914) 437.

[5] H. Kolsky, An Investigation of the Mechanical Properties of Materials at Very High Rates of Loading, Proc. Phys. Soc. London 62b (1949) 676-700.

[6] M. A. Sutton, S. R. McNeill, J. D. Helm and Y. J. Chao, Advances in TwoDimensional and Three-Dimensional Computer Vision, in: Photomechanics, P. K. Rastogi, eds., (Springer, Berlin (Germany), 2000), Topics in Appl. Phys., $77323-372$.

[7] M. A. Sutton, J.-J. Orteu and H. Schreier, Image correlation for shape, motion and deformation measurements: Basic Concepts, Theory and Applications, (Springer, New York, NY (USA), 2009).

[8] V. Tiwari, M. A. Sutton and S. R. McNeill, Assessment of High Speed Imaging Systems for 2D and 3D Deformation Measurements: Methodology Development and Validation, Exp. Mech. 47 [4] (2007) 561-579.

[9] F. Barthelat, Z. Wu, B. C. Prorok and H. D. Espinosa, Dynamic torsion testing of nanocrystalline coatings using high-speed photography and digital image correlation, Exp. Mech. 43 [3] (2003) 331-340. 
[10] I. Elnasri, S. Pattofatto, H. Zhao, H. Tsitsiris, F. Hild and Y. Girard, Shock enhancement of cellular structures under impact loading: Part I Experiments, J. Mech. Phys. Solids 55 (2007) 2652-2671.

[11] A. Gilat, T. Schmidt and J. Tyson, Full field measurement during a tensile split Hopkinson bar experiment, J. Phys. IV 134 (2006) 687-692.

[12] G. Besnard, S. Guérard, S. Roux and F. Hild, A space-time approach in digital image correlation: Movie-DIC, Optics Lasers Eng. 49 (2011) 71-81.

[13] M. S. Kurigulige, H. V. Tippur and T. S. Denney, Measurement of transient deformation using digital image correlation method and high-speed photography: application to dynamic fracture, Appl. Optics 46 [22] (2007) 50835096.

[14] V. Tiwari, M. A. Sutton, S. R. McNeill, S. Xua, X. Deng, W. L. Fourney and D. Bretall, Application of 3D image correlation for full-field transient plate deformation measurements during blast loading, Int. J. Impact Eng. 36 [6] (2009) 862-874.

[15] G. Besnard, J.-M. Lagrange, F. Hild, S. Roux and C. Voltz, Characterization of necking phenomena in high speed experiments by using a single camera, EURASIP J. Im. Video. Proc. 2010 [215956] (2010) 15 p.

[16] http://www.techimaging.com/.

[17] F. Hild, B. Raka, M. Baudequin, S. Roux and F. Cantelaube, Multi-Scale Displacement Field Measurements of Compressed Mineral Wool Samples by Digital Image Correlation, Appl. Optics IP 41 [32] (2002) 6815-6828.

[18] O. Faugeras, Three-dimensional computer vision, a geometric viewpoint, (MIT Press, Cambridge (USA), 1993).

[19] R. Y. Tsai, A versatile camera calibration technique for high-accuracy 3D machine vision metrology using off-the-shelf tv cameras and lenses, J. Robotics 
Autom. RA-3 (1987) 323-343.

[20] G. Besnard, F. Hild and S. Roux, "Finite-element" displacement fields analysis from digital images: Application to Portevin-Le Chatelier bands, Exp. Mech. 46 (2006) 789-803.

[21] L. Chevalier, S. Calloch, F. Hild and Y. Marco, Digital Image Correlation used to Analyze the Multiaxial Behavior of Rubber-Like Materials, Eur. J. Mech. A/Solids 20 (2001) 169-187.

[22] G. Besnard, Caractérisation et quantification de surfaces par stéréocorrélation pour des essais mécaniques du quasi statique à la dynamique ultra-rapide, (PhD thesis, ENS de Cachan, 2010).

[23] U. S. Lindholm, R. L. Bessy and G. V. Smith, Effect of strain rate on yield strength tensile strength and elongation of three aluminium alloy, J. Mat. 6 (1971) 119-133.

[24] N. F. Mott, Fragmentation of Shell Cases, Proc. Roy. Soc. London A189 (1947) 300-308.

[25] T. von Karman and P. Duwez, The propagation of plastic deformation in solids, J. Appl. Phys. 21 (1950) 987-994.

[26] G. I. Taylor, The Scientific Papers of G. I. Taylor, (Cambridge University Press, London, 1958).

[27] G. Besnard, H. Leclerc, S. Roux and F. Hild, Analysis of Image Series through Digital Image Correlation, J. Strain Analysis [accepted] (2012). 


\section{Appendix}

Lagrange's diagram of the Hopkinson bar setup (Figure 2) is shown in Figure 16. For all the tested geometries, a first loading ramp is followed by a plateau and a subsequent ramp before failure is observed. For the larger samples, a third ramp was needed. However, the results reported herein stop at the end of the second ramp (see shaded area). From Figure 16, it can be concluded that the reflected signal coming from the transmitter bar has not reached the sample.

The following analysis is carried out by assuming that the impedance of the sample $Z_{s}$ is very small compared to that of the bars $Z$. The reflections on the free surface of the transmitter bar are not accounted for since they do not alter significantly the various states in the incident bar. The stress and velocity states are denoted by roman numbers in Figure 2. Let $-V$ denote the velocity of the striker.

- State $\mathrm{I}$ is characterized by $V_{I}=-V / 2$ and $\sigma_{I}=Z V / 2$. In that case, the incident bar is in tension.

- For state II, $V_{I I}=0$ and $\sigma_{I I}=0$. The striker is now motionless.

- State III is characterized by $V_{I I I} \approx-V$ and $\sigma_{I I I} \approx-Z_{s} V$. The velocity of the interface between the incident bar and the sample is approximately equal to the initial velocity of the striker. The sample is loaded in tension.

- For state IV, $V_{I V} \approx-V / 2$ and $\sigma_{I V} \approx-Z V / 2$. The incident bar is in compression.

- State $\mathrm{V}$ is characterized by $V_{V} \approx 0$ and $\sigma_{V} \approx 0$. The sample is no longer loaded in tension.

- For state VI, $V_{V I} \approx-V$ and $\sigma_{V I} \approx 0$. The incident bar moves again. At 
this stage the striker and the incident bar are no longer in contact.

- State VII is characterized by $V_{V I I} \approx-V / 2$ and $\sigma_{V I I} \approx Z V / 2$. The incident bar is in tension again, but with stress and velocity levels less than those observed in state I.

- State VIII, which is similar to state III, corresponds to a new tensile loading of the sample.

- Similarly, state IX is very close to states II and V. 


\section{List of Tables}

1 Experimental information of the four tests on cylinders.

2 Experimental information of the four tests on parallelepipeds.

25

3 Experimental results on cylindrical samples.

4 Experimental results on parallelepipedic samples. 
Table 1

Experimental information of the four tests on cylinders.

\begin{tabular}{|c||c|c|c|c|}
\hline Test & A & B & C & D \\
\hline Gauge length $(\mathrm{mm})$ & 10 & 20 & 30 & 40 \\
Striker velocity $(\mathrm{m} / \mathrm{s})$ & 18.7 & 24.2 & 26.6 & 26.6 \\
Picture definition (pixels) & $384 \times 96$ & $640 \times 80$ & $768 \times 64$ & $896 \times 64$ \\
& 52 & 63 & 78 & 78 \\
Physical size of 1 pixel $(\mu \mathrm{m})$ & 52,500 & 50,000 & 52,500 & 45,000 \\
Acquisition rate $(\mathrm{fps})$ & 4.0 & 4.1 & 3.9 & 4.0 \\
\hline Integration time $(\mu \mathrm{s})$ & & & & \\
\hline
\end{tabular}

Table 2

Experimental information of the four tests on parallelepipeds.

\begin{tabular}{|c|c|c|c|c|}
\hline Test & $\mathrm{E}$ & $\mathrm{F}$ & G & $\mathrm{H}$ \\
\hline Gauge length (mm) & 10 & 20 & 30 & 40 \\
\hline Striker velocity $(\mathrm{m} / \mathrm{s})$ & 19.1 & 24.2 & 26.7 & 26.7 \\
\hline Picture definition (pixels) & $384 \times 112$ & $512 \times 96$ & $570 \times 80$ & $768 \times 80$ \\
\hline Physical size of 1 pixel $(\mu \mathrm{m})$ & 71 & 83 & 80 & 80 \\
\hline Acquisition rate (fps) & 50,000 & 50,000 & 50,000 & 45,000 \\
\hline Integration time $(\mu \mathrm{s})$ & 4.0 & 4.0 & 4.1 & 3.9 \\
\hline
\end{tabular}


Table 3

Experimental results on cylindrical samples.

\begin{tabular}{|c||c|c|c|c|}
\hline Test & A & B & C & D \\
\hline Strain rate $\left(\mathrm{s}^{-1}\right)$ & $1600 \pm 100$ & $1300 \pm 200$ & $800 \pm 150$ & $600 \pm 70$ \\
Neck length $(\mathrm{mm})$ & 1.7 & 1.3 & 2.3 & 1.8 \\
Onset strain & 0.18 & 0.3 & 0.25 & 0.2 \\
\hline
\end{tabular}

Table 4

Experimental results on parallelepipedic samples.

\begin{tabular}{|c||c|c|c|c|}
\hline Test & E & F & G & H \\
\hline Strain rate $\left(\mathrm{s}^{-1}\right)$ & $1400 \pm 270$ & $1000 \pm 140$ & $800 \pm 80$ & $600 \pm 100$ \\
Neck length $(\mathrm{mm})$ & 1.8 & 2.7 & 2.4 & 2.6 \\
Onset strain & 0.15 & 0.15 & 0.12 & 0.2 \\
\hline
\end{tabular}




\section{List of Figures}

1 Experimental setup used to monitor necking by using two high speed cameras (a). The red circle marks the location of the tested sample. Aluminum samples to be tested in tension with split Hopkinson pressure bars (b).

2 Principle of the tensile setup of split Hopkinson pressure bars used herein.

3 Projection of points of interest on an image pair of sample A.

4 Visualization of the maximum angle of the reconstructed shape prior to (sector delimited by continuous lines) and after (sector delimited by dotted lines) correction for sample A.

5 Contour detection (red lines) in the reference (a) and deformed (b) configurations of sample A.

6 Effect of pre-correction for temporal registration (sample A).

7 Pictures of the left camera when the necks are formed, and corresponding reconstructed surface for the four cylindrical samples. The color map corresponds to the radius.

8 Pictures of the right camera when the necks are formed, and corresponding longitudinal strain field for the four parallelepipedic samples. 
9 Radius as a function of longitudinal coordinate along the gauge length for the four cylindrical samples. The picture number is indicated.

10 Mean major principal strain as a function of time for the four cylindrical samples. The analysis of these data yields the strain rate reported in Table 3 for the four tested samples.

11 Mean major principal strain as a function of time for the four parallelepipedic samples. The analysis of these data yields the strain rate reported in Table 4 for the four tested samples.

12 Major principal strain as a function of longitudinal coordinate along the gauge length for the four cylindrical samples. The picture number is indicated. The dotted lines depict the zones in which average strains in the necks are determined.

13 Major principal strain as a function of longitudinal coordinate along the gauge length for the four parallelepipedic samples. The picture number is indicated. The dotted lines depict the zones in which average strains in the necks are determined.

14 Mean major principal strain as a function of time for the four cylindrical samples. The solid line corresponds to the average value of the whole gauge area, and the dashed lines is the average in the necks. 
15 Mean major principal strain as a function of time for the four parallelepipedic samples. The solid line corresponds to the average value of the whole gauge area, and the dashed lines is the average in the necks.

16 Lagrange diagram for the setup used herein. The gray shaded area shows the time interval during which the pictures are analyzed herein. 


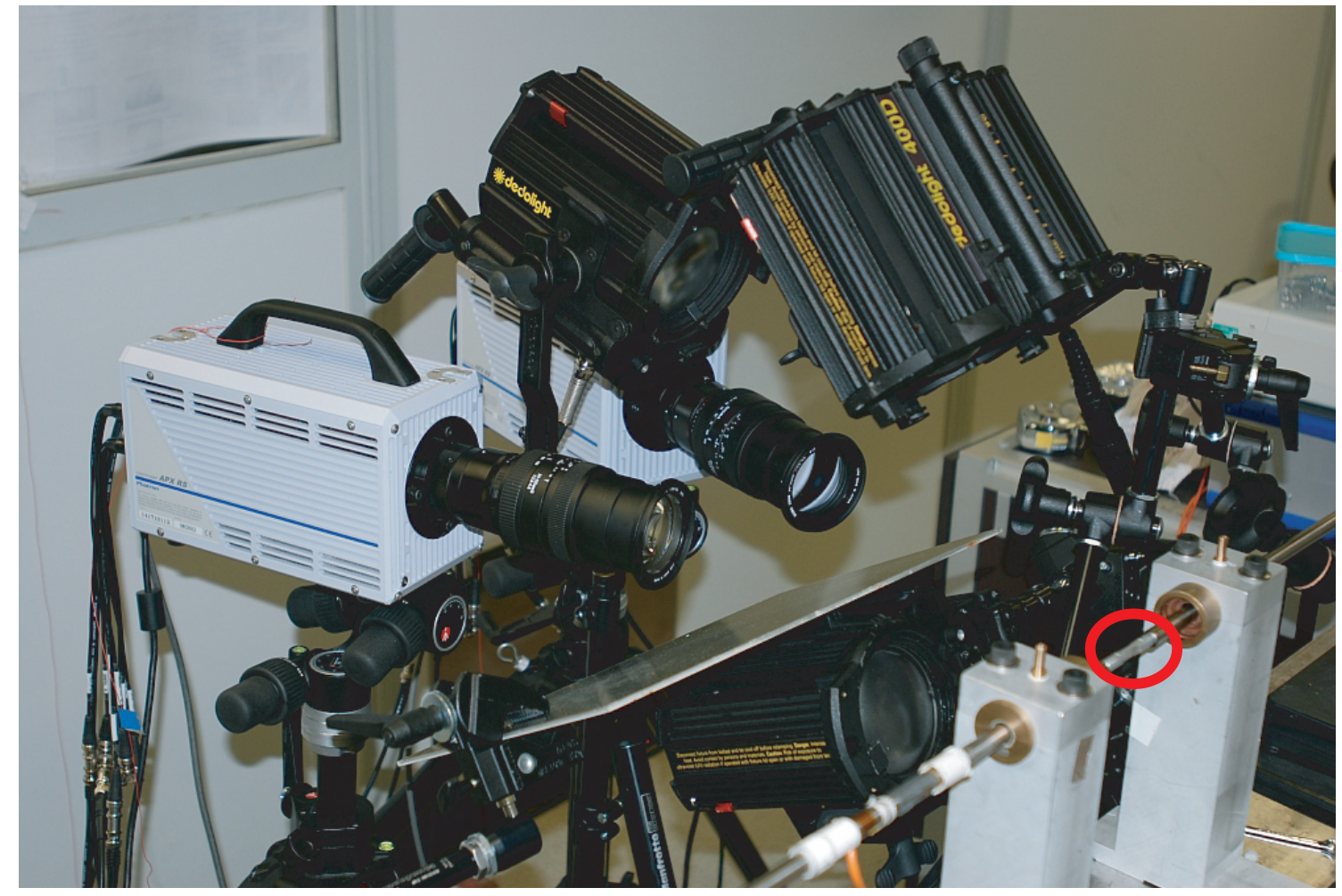

(a)

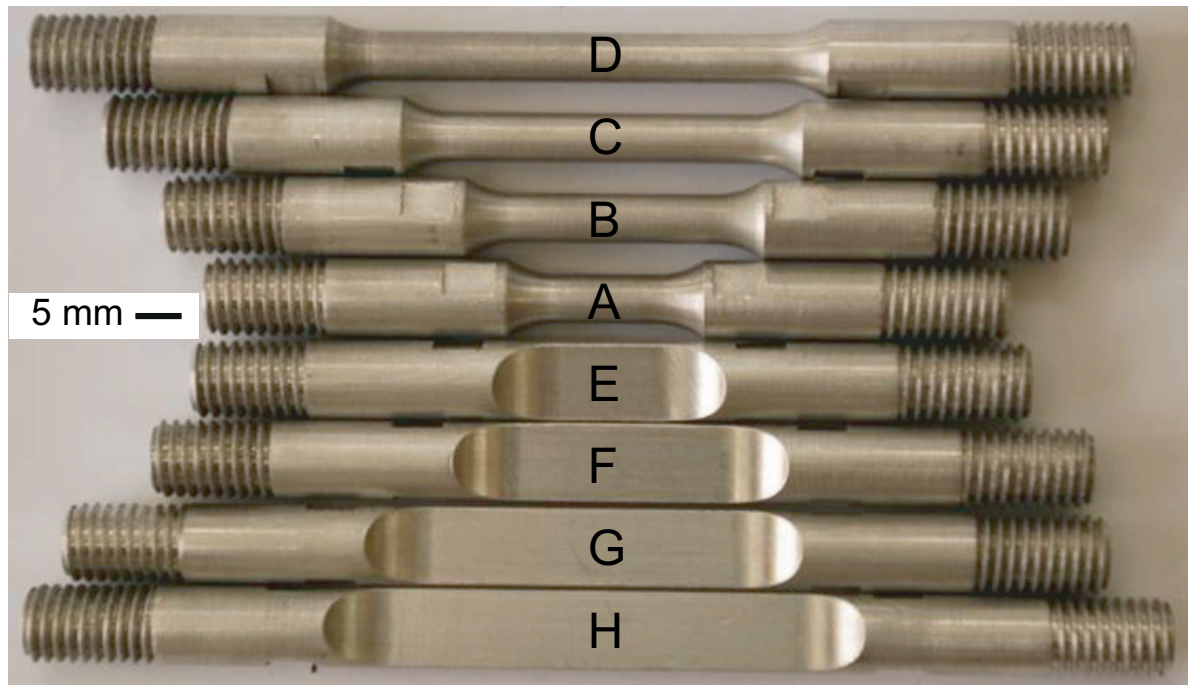

(b)

Fig. 1. Experimental setup used to monitor necking by using two high speed cameras (a). The red circle marks the location of the tested sample. Aluminum samples to be tested in tension with split Hopkinson pressure bars (b). 


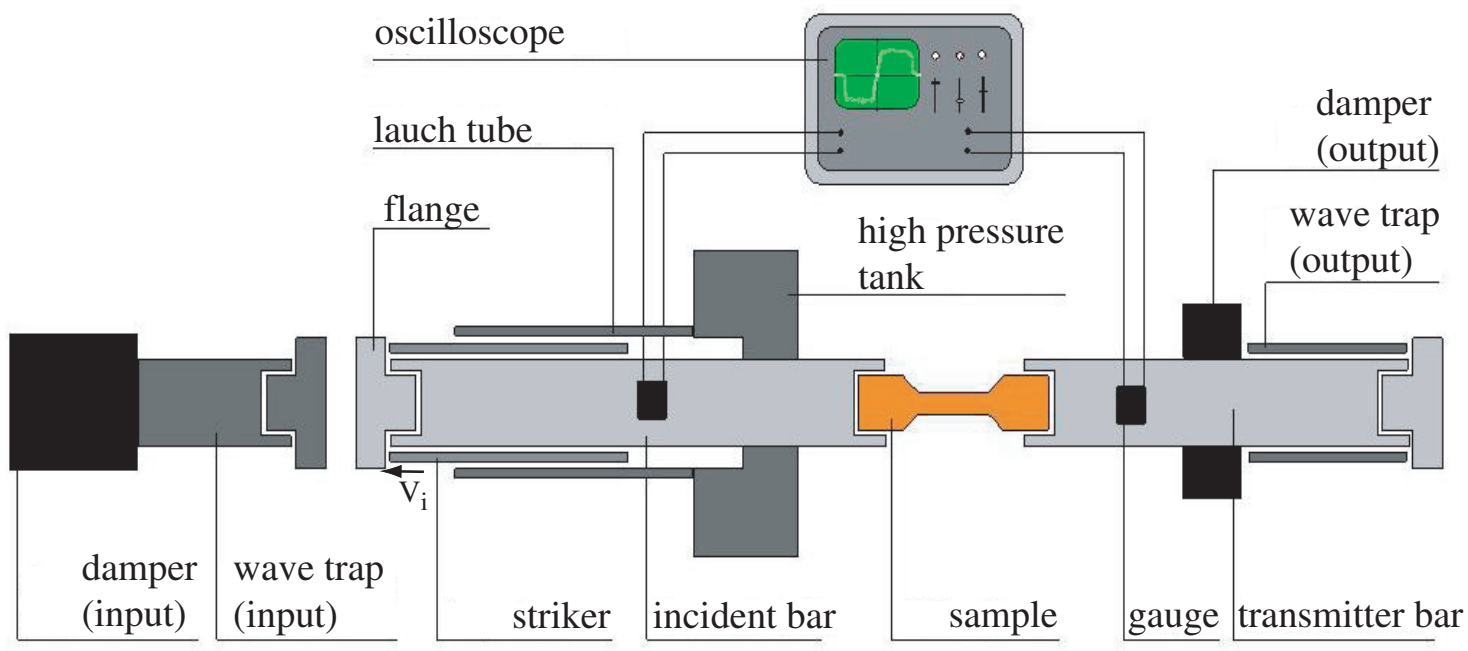

Fig. 2. Principle of the tensile setup of split Hopkinson pressure bars used herein. 


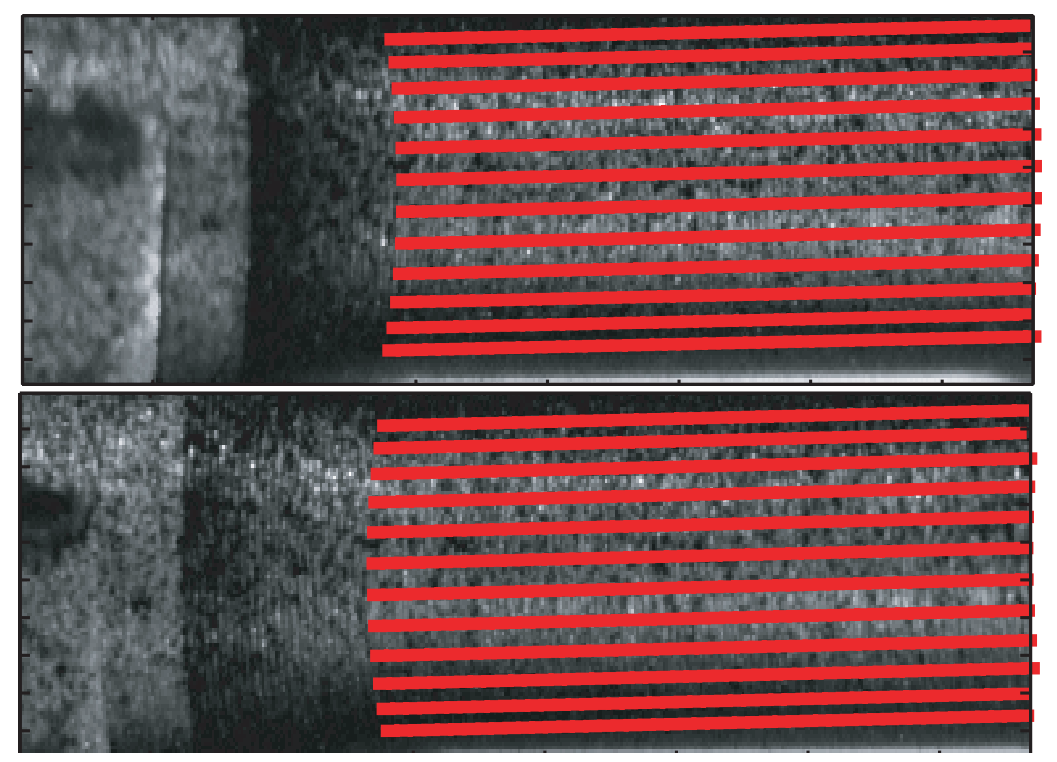

Fig. 3. Projection of points of interest on an image pair of sample A. 


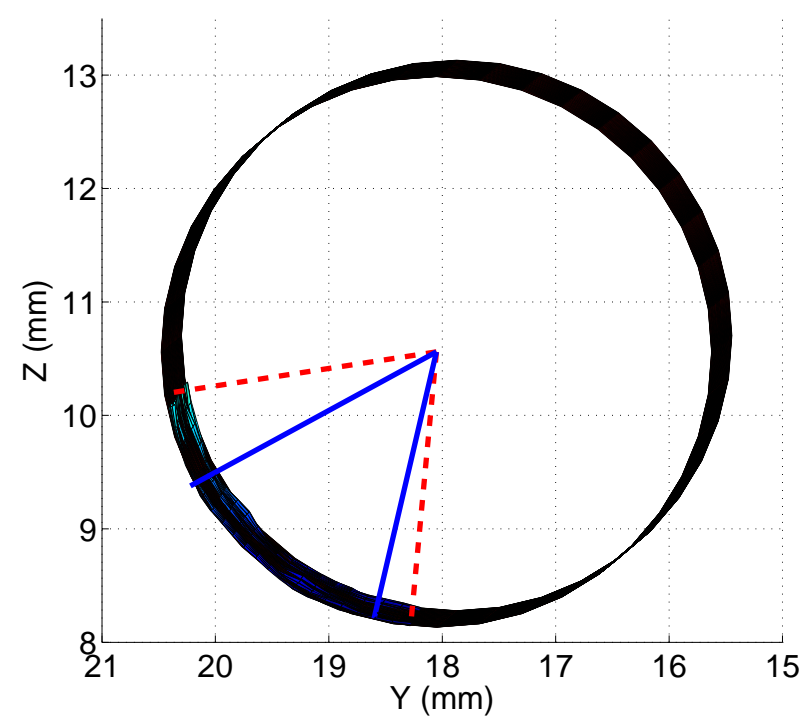

Fig. 4. Visualization of the maximum angle of the reconstructed shape prior to (sector delimited by continuous lines) and after (sector delimited by dotted lines) correction for sample A. 


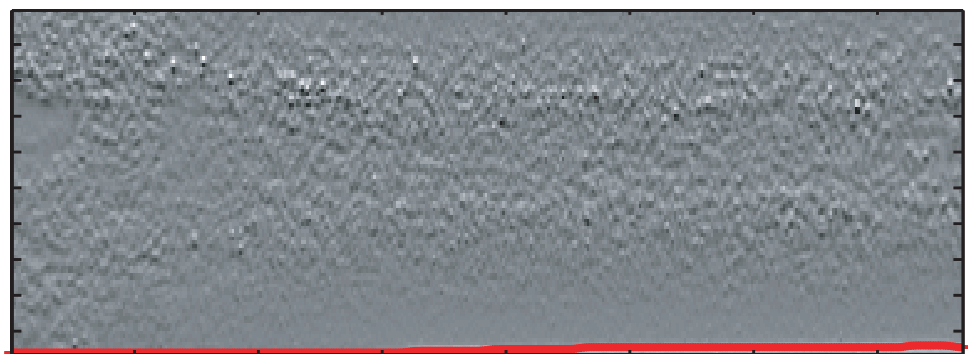

(a)

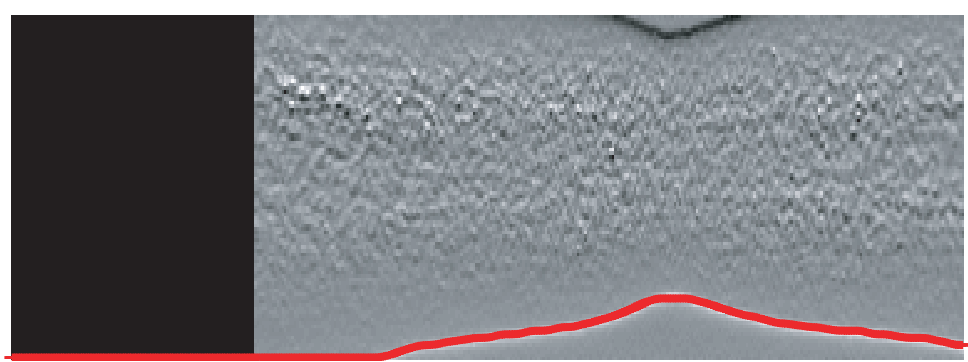

(b)

Fig. 5. Contour detection (red lines) in the reference (a) and deformed (b) configurations of sample A. 


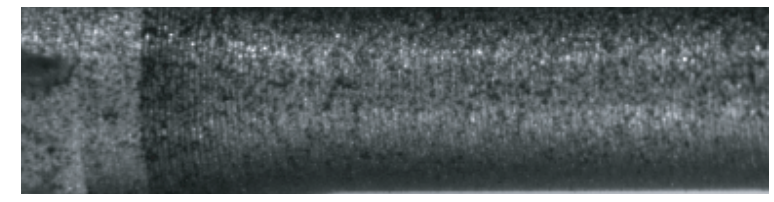

(a) Reference picture

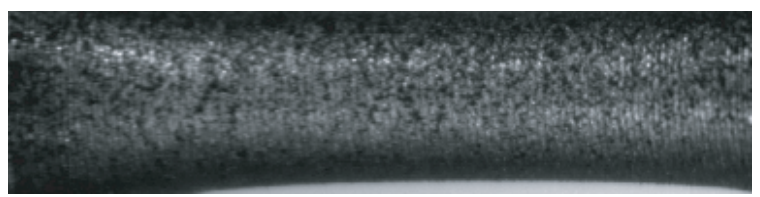

(c) Deformed picture

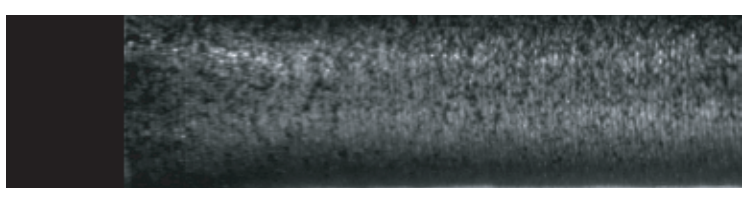

(e) Deformed picture pre-corrected with the estimated displacement field

Fig. 6. Effect of pre-correction for temporal registration (sample A).
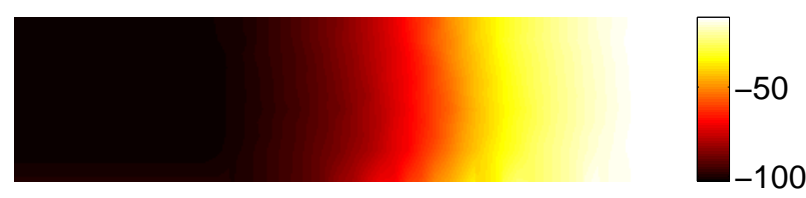

(b) Longitudinal displacement field (in pixels)

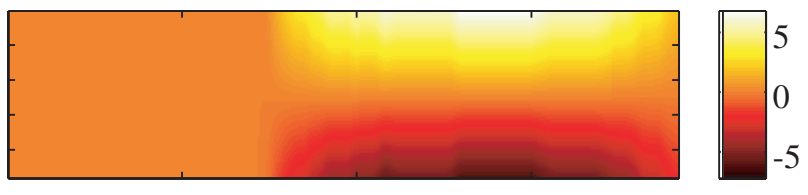

(d) Transverse displacement field (in pixels) 


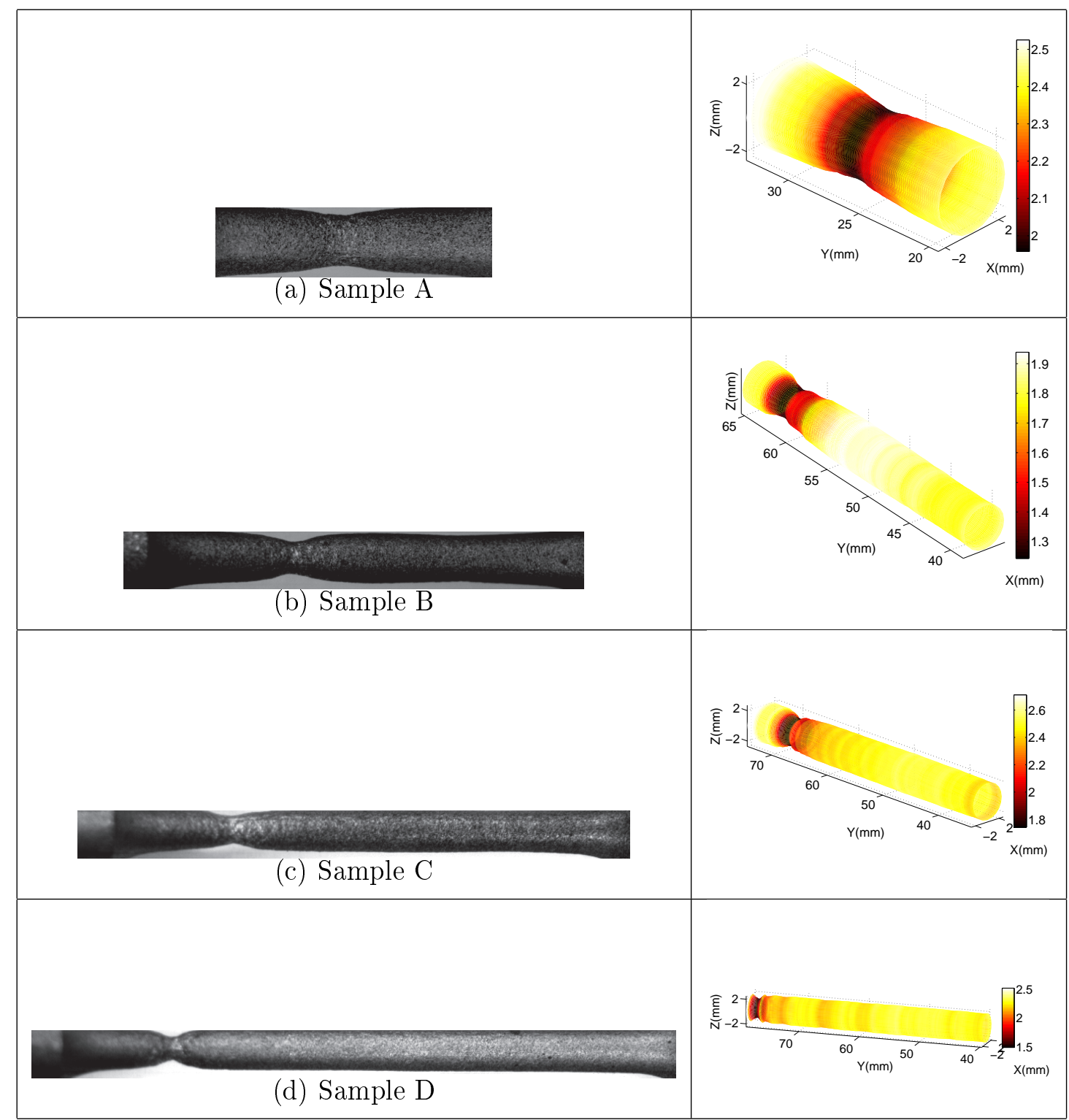

Fig. 7. Pictures of the left camera when the necks are formed, and corresponding reconstructed surface for the four cylindrical samples. The color map corresponds to the radius. 


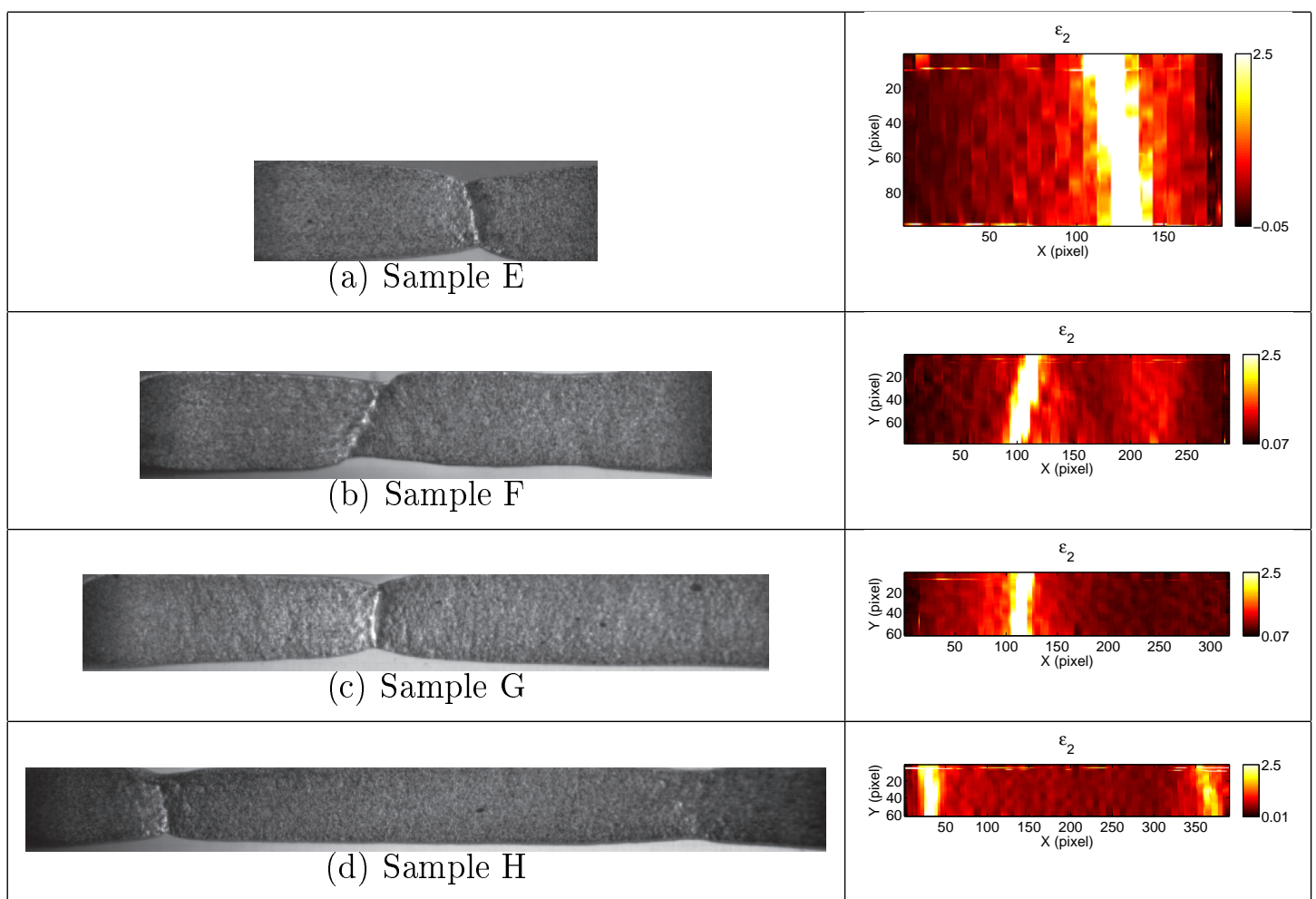

Fig. 8. Pictures of the right camera when the necks are formed, and corresponding longitudinal strain field for the four parallelepipedic samples. 


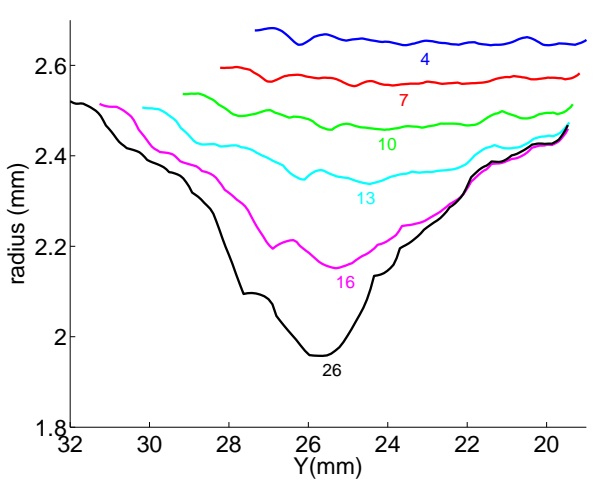

(a) Sample A

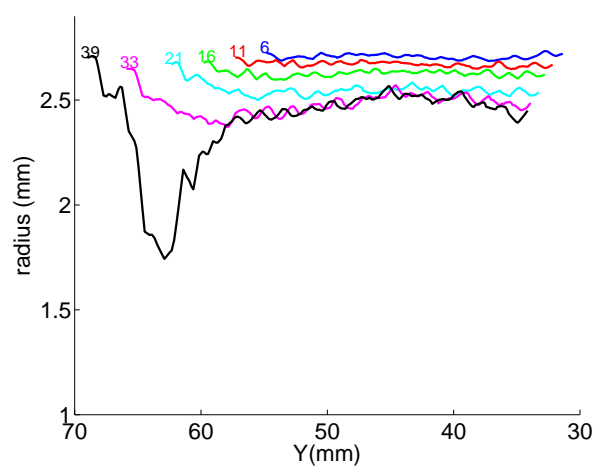

(c) Sample C

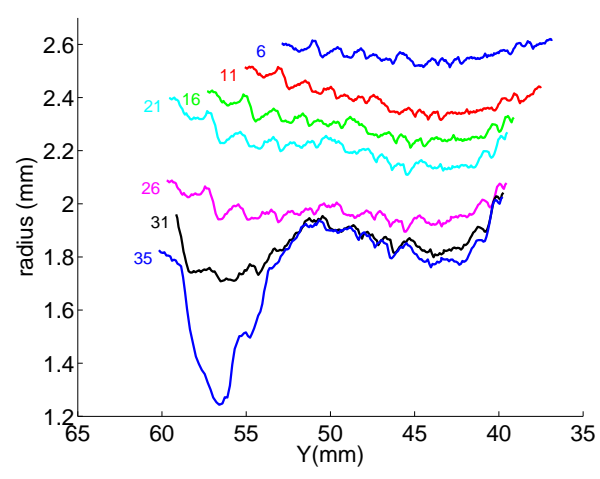

(b) Sample B

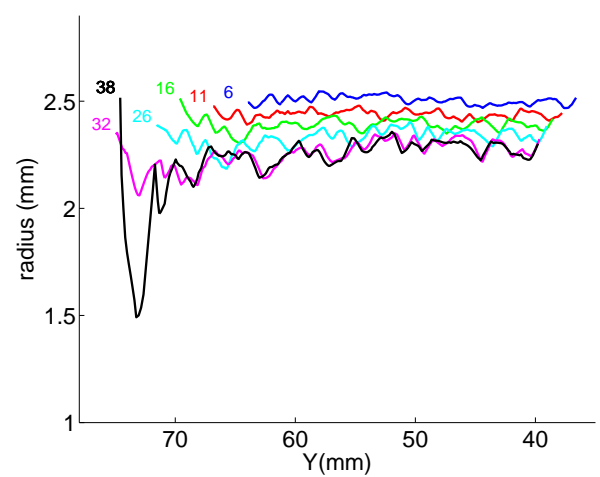

(d) Sample D

Fig. 9. Radius as a function of longitudinal coordinate along the gauge length for the four cylindrical samples. The picture number is indicated. 


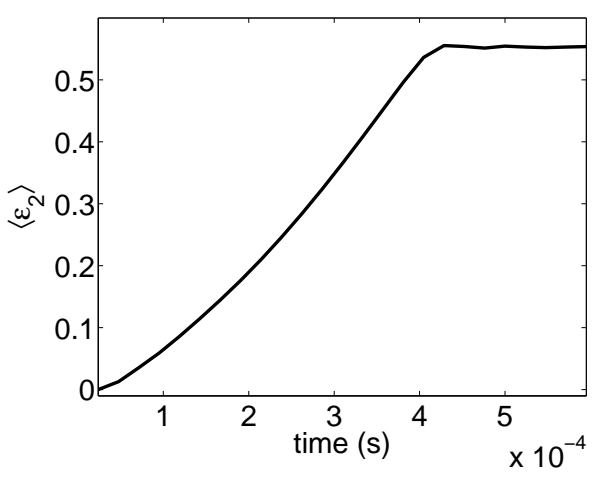

(a) Sample A

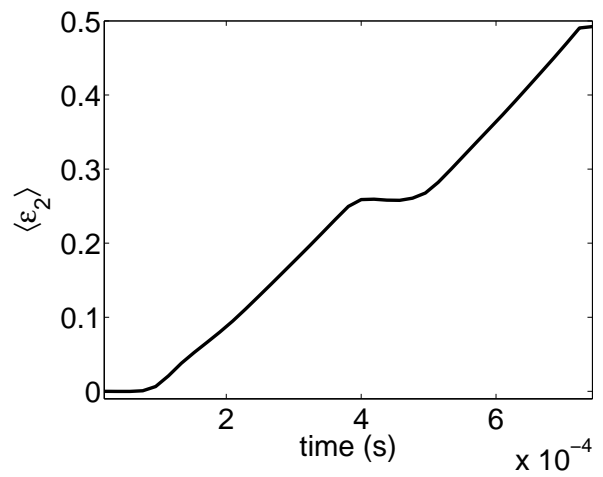

(c) Sample C

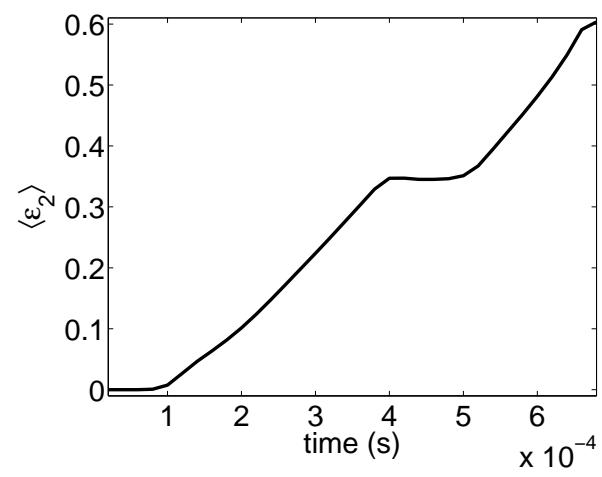

(b) Sample B

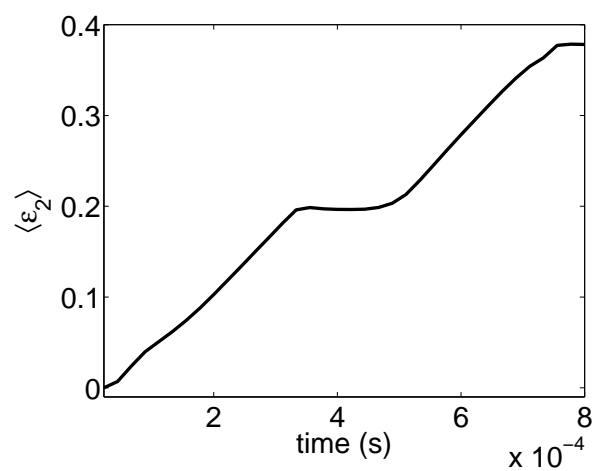

(d) Sample D

Fig. 10. Mean major principal strain as a function of time for the four cylindrical samples. The analysis of these data yields the strain rate reported in Table 3 for the four tested samples. 


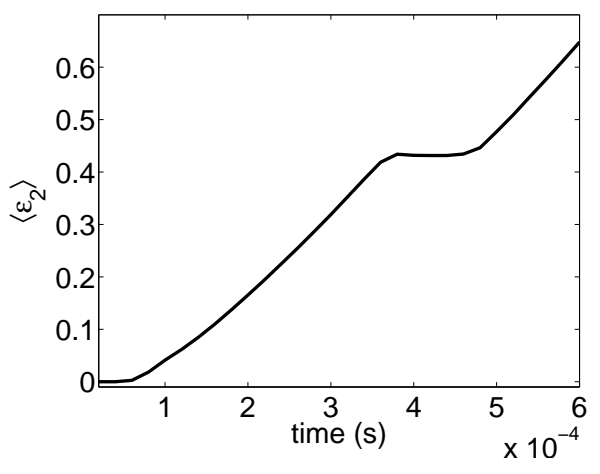

(a) Sample E

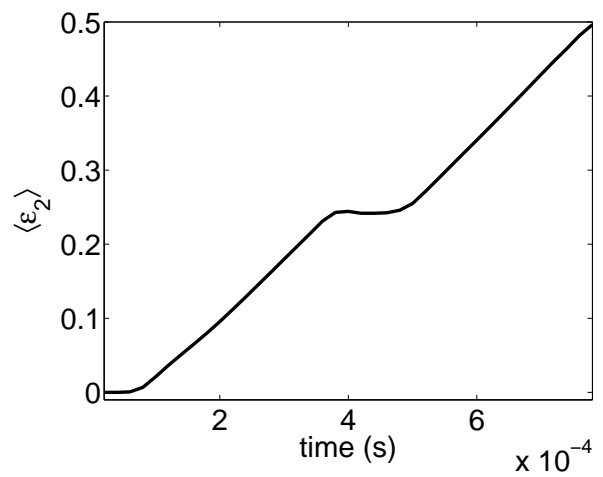

(c) Sample G

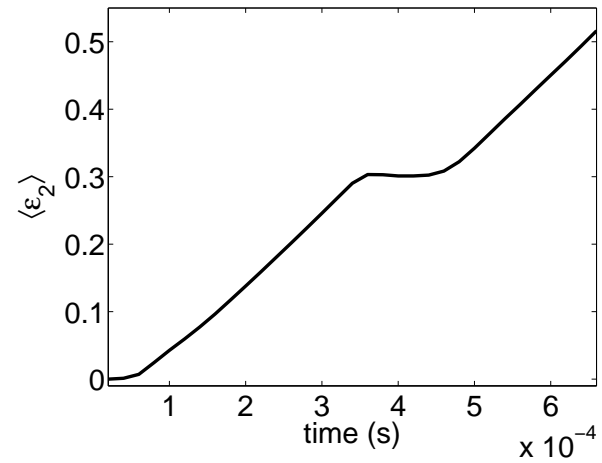

(b) Sample F

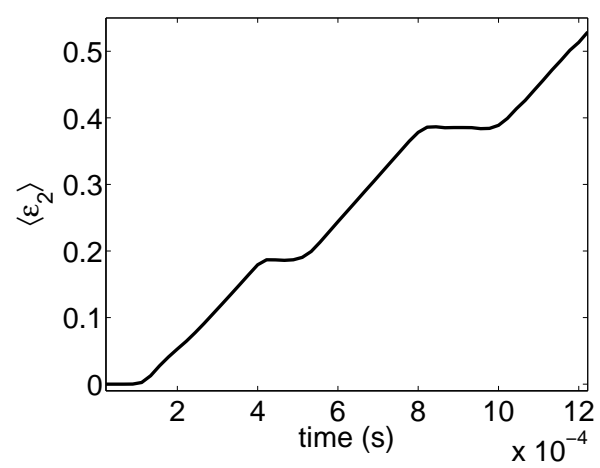

(d) Sample H

Fig. 11. Mean major principal strain as a function of time for the four parallelepipedic samples. The analysis of these data yields the strain rate reported in Table 4 for the four tested samples. 


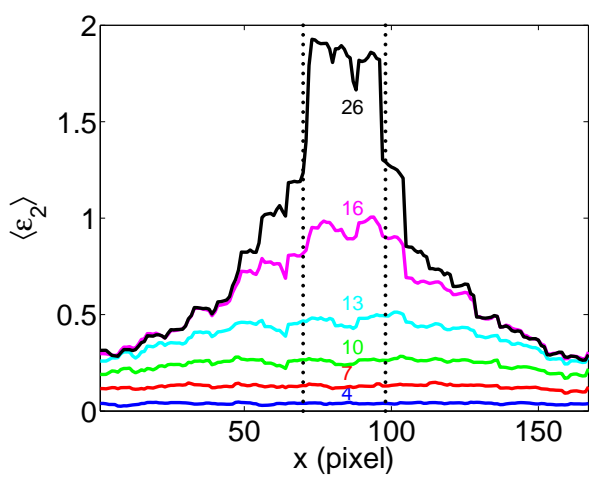

(a) Sample A

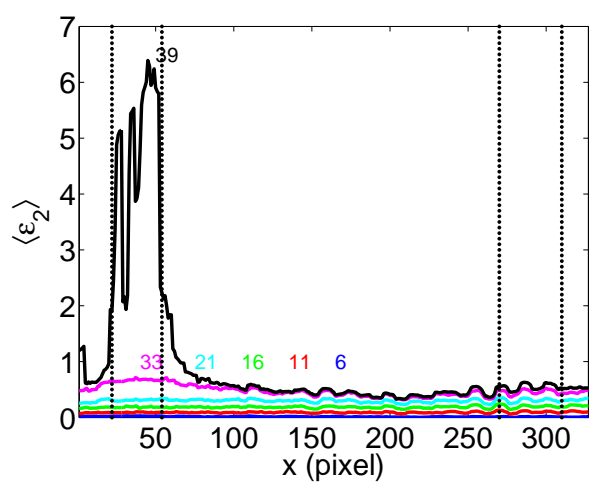

(c) Sample C

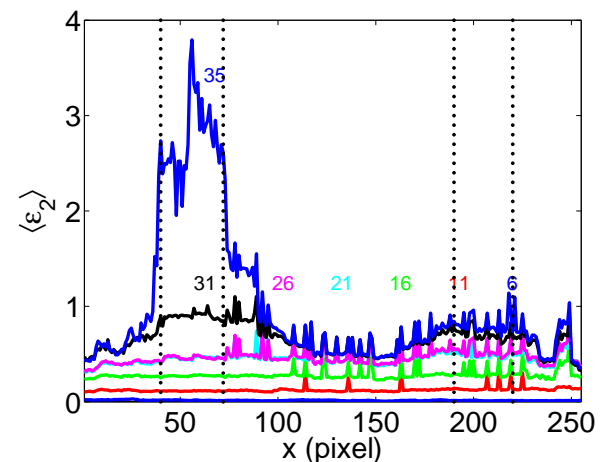

(b) Sample B

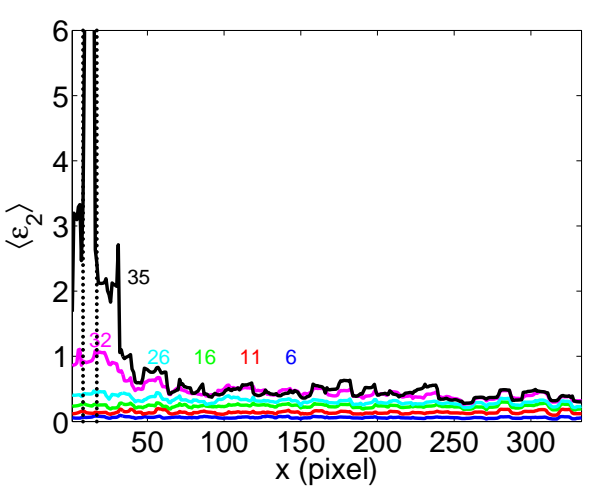

(d) Sample D

Fig. 12. Major principal strain as a function of longitudinal coordinate along the gauge length for the four cylindrical samples. The picture number is indicated. The dotted lines depict the zones in which average strains in the necks are determined. 


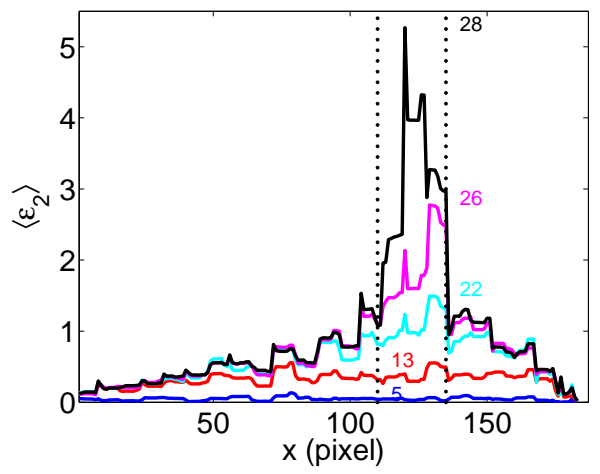

(a) Sample E

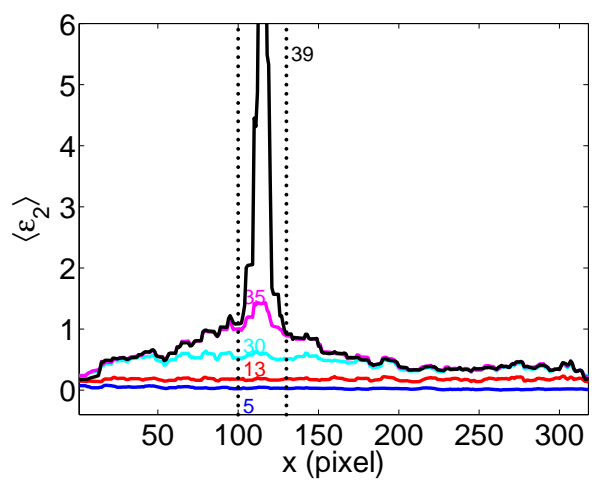

(c) Sample G

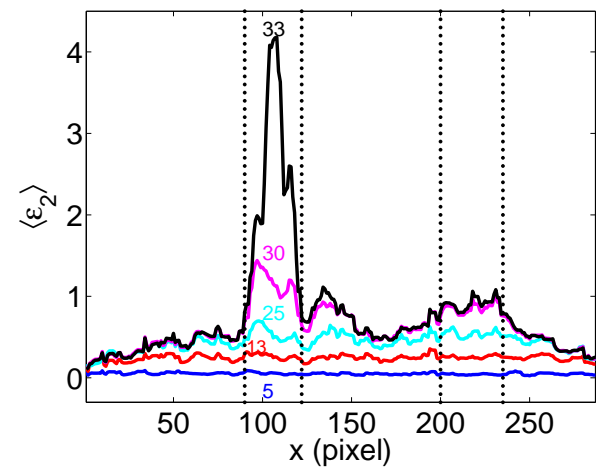

(b) Sample F

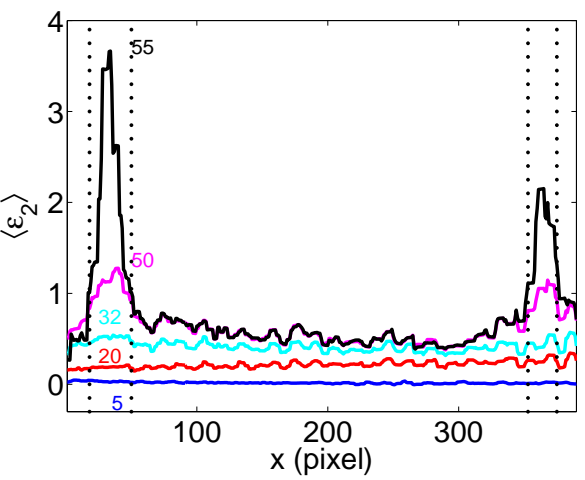

(d) Sample H

Fig. 13. Major principal strain as a function of longitudinal coordinate along the gauge length for the four parallelepipedic samples. The picture number is indicated. The dotted lines depict the zones in which average strains in the necks are determined. 


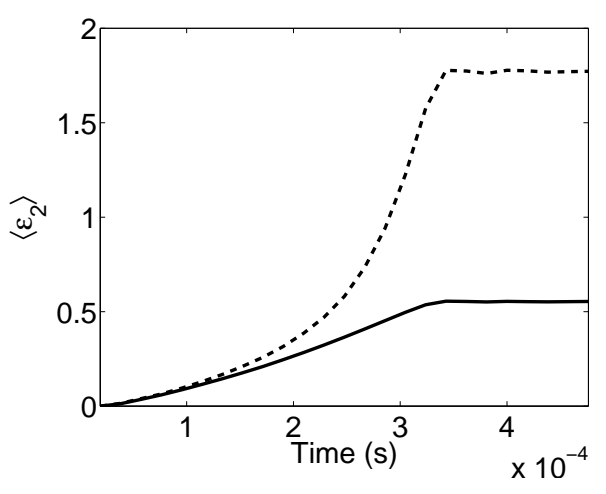

(a) Sample A

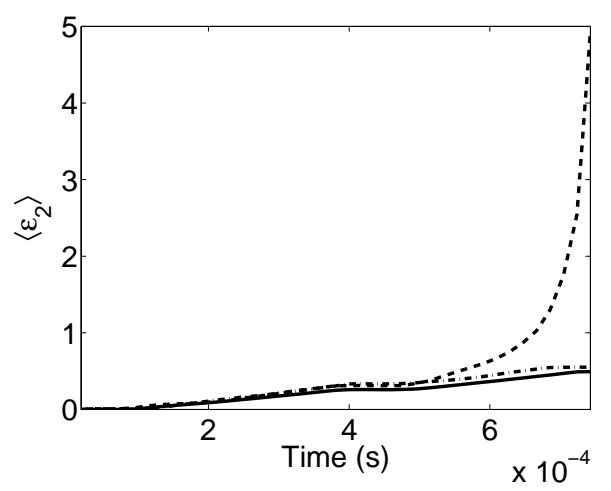

(c) Sample C

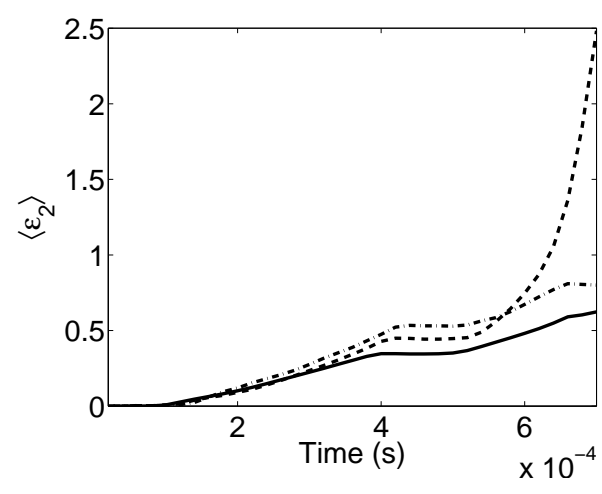

(b) Sample B

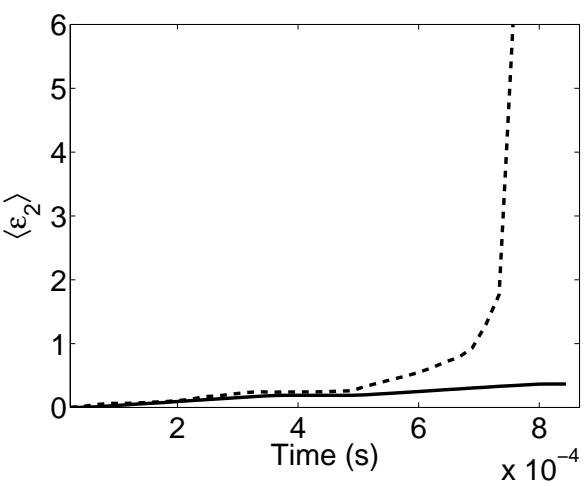

(d) Sample D

Fig. 14. Mean major principal strain as a function of time for the four cylindrical samples. The solid line corresponds to the average value of the whole gauge area, and the dashed lines is the average in the necks. 


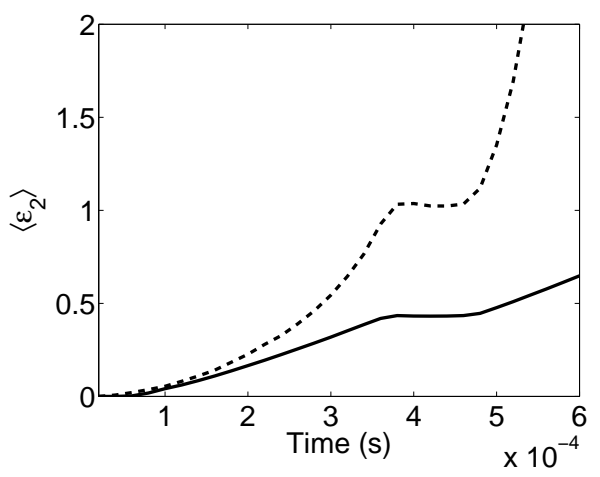

(a) Sample E

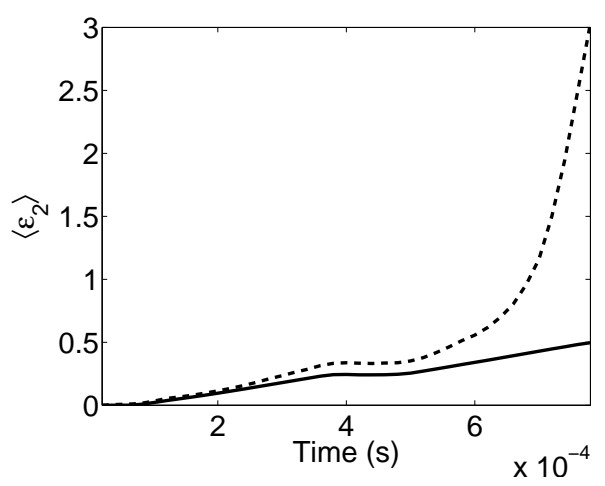

(c) Sample G

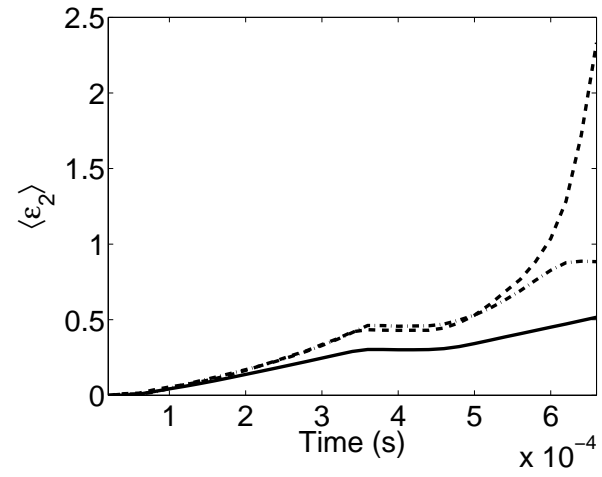

(b) Sample F

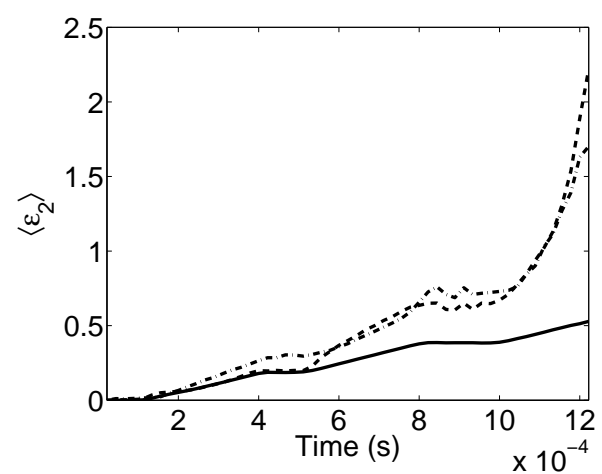

(d) Sample H

Fig. 15. Mean major principal strain as a function of time for the four parallelepipedic samples. The solid line corresponds to the average value of the whole gauge area, and the dashed lines is the average in the necks. 

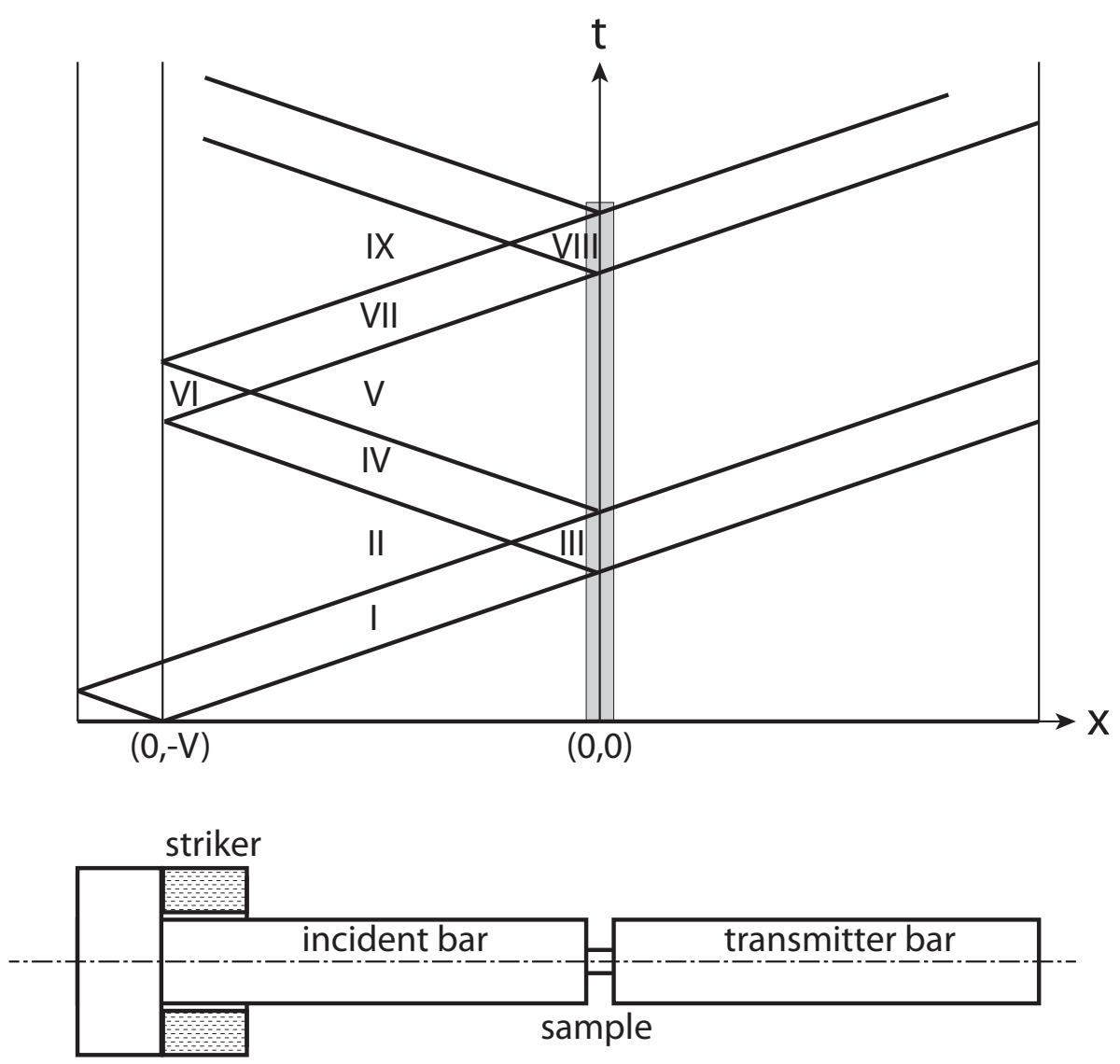

Fig. 16. Lagrange diagram for the setup used herein. The gray shaded area shows the time interval during which the pictures are analyzed herein. 